\title{
Dielectric functions and optical parameters of heavily doped and/or highly excited Si:P
}

\author{
MAREK BASTA $^{1,2^{*}}$, ZBIGNIEW T. KUŹNICKI $^{1}$ \\ ${ }^{1}$ Photonic Systems Laboratory, Pôle API Parc d'Innovation, \\ Boulevard Sébastien Brant, BP 10413, 67400 Illkirch, France \\ ${ }^{2}$ Institute of Physics, Wrocław University of Technology, \\ Wybrzeże Wyspiańskiego 27, 50-370 Wrocław, Poland \\ ${ }^{*}$ Corresponding author: marek.basta@pwr.wroc.pl
}

\begin{abstract}
Recently shown photonic and optoelectronic potentialities of Si-based materials and devices require an accurate representation for their optical functions. A predictive model of dielectric function for heavily doped and/or highly excited Si:P is presented. The influence of dopants and of free-carrier population has been calculated independently, allowing the determination of accuracy in usual approximations. The effect of Drude parameters on the heavily doped Si:P optical response is taken into account. All results are supported by experimental data.
\end{abstract}

Keywords: heavily doped $\mathrm{Si}$, strongly excited $\mathrm{Si}$, dielectric function, Drude-Lorentz approximation, optical model.

\section{Introduction}

Tabular representation of the dielectric response [1] of semiconductors [2, 3] is often sufficient for analyzing simple materials by optical methods, but it is usually insufficient when the attention is moved towards complex materials. Analytical representations $[4,5]$ in such cases are much more convenient, allowing rapid predictions and analysis of subtle property changes of a material. They become essential in the fabrication of new materials that exhibit different properties than their classical counterparts [6] and they also find use in quality control of epitaxial growth where constant monitoring of optical properties must take place [7]. Another important application lies in new generation of semiconductor devices [8], where very thin films are stacked together, for example in third generation multi-junction solar cells [9]. Existing solutions and theoretical models allow analyzing either the generic response of a given material for a broad range of energies [10] or accurate response for a very specific case and conditions [11], trading off generality for accuracy and usually [12] 
their increased complexity at the same time. Even so, many accurate models and theoretical approaches still working for studying single material fail when the emphasis is turned to a multi-material (composite) structure [13].

Specific optoelectronic properties of heavily doped and/or highly excited semiconductors, which differ in various ways from perfectly known (non-degenerated) materials, lead to new often unpredicted applications $[14,15]$. The properties of such materials find use and are appreciated in late generation electronic circuits, and will probably appear in future all-Si optical and photonic applications. Several effects have been discovered [16] in materials [17] to produce often unpredicted properties or enhancement of known properties [18, 19]. Despite extensive studies over the past twenty years, there are several non-elucidated questions about optical features [20, 21]. Results of the systematic investigation into differences among ellipsometrically measured pseudodielectric functions are also found in the literature [13].

Information about the dielectric functions and their critical points (CP) corresponding to interband transitions of such materials is necessary for fabrication and proper control of new materials [3]. Measurements of these interband transitions have been performed by several optical techniques, including reflectance [22, 23], ellipsometry $[24,25]$ and reflectance modulation techniques such as magnetoreflectance [26], thermoreflectance [27, 28], electroreflectance [29,30] and wavelength-modulated reflectance [31, 32].

Semiconductors are especially interesting due to an unusual number of physical phenomena and possible applications [33,34]. Many papers have been published covering the behavior of $\mathrm{CP}$ and dielectric functions for $\mathrm{Si}[35,36]$ and other semiconductors [37, 38] as a function of illumination [39, 40], temperature [41, 42], composition of the material [43,44] or its phase [45, 46], method of preparation [47], or size [48]. Various analytical methods $[49,50]$ exist that can be used to predict (or estimate) the quantities in a given range of energy [51,52]. The quantities are, e.g., the dielectric function of a material [53] with a dense free-electron gas [54,55], composite medium [56] or metamaterial [4]. The methods used are: the density functional theory [57], exchange-correlation potential [58], k.p method [59,60], tight-binding approximation $[61,62]$ or parabolic band approximation $[63,64]$, to name just a few.

Extremely heavily doped $\mathrm{Si}$ or highly excited $\mathrm{Si}$, or the $\mathrm{Si}$ that is less heavily doped and/or highly excited show optoelectronic properties that differ in various ways from the perfectly known non-degenerate and/or weakly excited material. Such a situation sometimes offers unpredicted applications [6] that find their place in late generation electronic circuits and will appear in the future all-Si optoelectronics and photonics. Despite extensive studies over the past twenty years, there are several non-elucidated questions which for example appear in a systematic investigation into ellipsometrically measured pseudodielectric functions [13].

We have focused in particular on Si:P (moderately and heavily P-doped crystalline $\mathrm{Si}$ ) and highly excited $\mathrm{Si}$ (containing an extremely dense free-carrier gas) looking for general tendencies of the dielectric functions of a system. The results are directly 
useful in the design, manufacturing and control of newly fabricated materials and devices $[3,36]$ by allowing a prediction of functioning and yield $[4,58,65]$. New fabrication techniques permit extremely heavy $\mathrm{Si}: \mathrm{P}$ doping, more than $N_{d}=$ $=2.3 \times 10^{21} \mathrm{~cm}^{-3}$, which exceeds the solid state solubility of P in Si at $T=300 \mathrm{~K}$.

The general behavior of the dielectric function under small doping variation was studied in [1]. But any consistent model for describing the whole doping range, from intrinsic Si to extremely heavily doped Si (at the limit of the solubility), does not exist to date. In our investigations we used as a reference the experimental data for undoped $\mathrm{Si}[2]$ and for heavily doped Si (Si:P) [66].

Usually behavior variations resulting from doping or from generated/injected carriers are treated in the same way. A femtosecond excitation of intrinsic Si (by injection of a free-carrier population) has shown that the corresponding dielectric functions change differently [67]. Thus, the problem becomes essential in design and manufacturing of devices containing a dense free-carrier population.

Our study, which is based on the theoretical approach of [11] and on the data of $[1-3,68]$, confirms that the changes of dielectric function caused by injected carriers or by heavily doping are not the same. Questions about the sensitivity of the nonlinear dielectric functions to the carrier-carrier interactions are still open.

\section{Theory and computational methodology}

\subsection{Harmonic oscillator approximation and Drude approach}

We propose a method of predicting dielectric functions of heavily doped $\mathrm{Si}(\mathrm{Si}: \mathrm{P})$ and heavily doped Si under high excitation (e.g., by incident light) that both lead to a dense additional/extrinsic free-carrier population. The well-known harmonic oscillator (HMO) approximation has been extensively studied over the years and some classical problems and disagreements between experimental data for Si and theory have been identified, like an inaccurate representation of the absorption edge associated with the direct bandgap [4, 5, 7-13,65-67]. Simple extensions to the classical model might solve the problem, but they lead to the loss of generality of the model itself [10-12]. For analyzing dielectric functions in materials that vary little, one might need a tool that is suited for a particular application and spectral range, rather than more generic but less accurate one. Such extensions have been developed for intrinsic Si and Si-derived materials in the past $[11,12]$, and they work well when parameterization of a given dielectric function is performed, but they are not capable of predicting optical properties when structural changes occur. For structural changes, a model equation introduced by ASPNES group [11] was expanded by the addition of a possible functional dependence of its parameters in regard to changing doping density, and later combined with a Drude extension that leads to the following set of equations:

$$
\varepsilon\left(\omega, N_{d}\right)=\varepsilon_{\infty}+\sum_{j} \frac{C_{0 j}\left(N_{d}\right)}{\omega^{2}}(A+B-C-D)-E
$$


where:

$$
\begin{aligned}
& A=\exp \left[i \beta_{j}\left(N_{d}\right)\right]\left[\omega_{g j}\left(N_{d}\right)-\omega-i \Gamma_{j}\left(N_{d}\right)\right]^{\mu_{j}\left(N_{d}\right)} \\
& B=\exp \left[-i \beta_{j}\left(N_{d}\right)\right]\left[\omega_{g j}\left(N_{d}\right)+\omega+i \Gamma_{j}\left(N_{d}\right)\right]^{\mu_{j}\left(N_{d}\right)} \\
& C=2 \operatorname{Re}\left\{\exp \left[-i \beta_{j}\left(N_{d}\right)\right]\left[\omega_{g j}\left(N_{d}\right)+i \Gamma_{j}\left(N_{d}\right)\right]^{\mu_{j}\left(N_{d}\right)}\right\} \\
& D=2 i \mu_{j}\left(N_{d}\right) \omega \operatorname{Im}\left\{\exp \left[-i \beta_{j}\left(N_{d}\right)\right]\left[\omega_{g j}\left(N_{d}\right)+i \Gamma_{j}\left(N_{d}\right)\right]^{\mu_{j}\left(N_{d}\right)-1}\right\} \\
& E=\frac{N_{\mathrm{e}-\mathrm{h}} e^{2}}{\varepsilon_{0} m_{\mathrm{opt}} m_{e} \omega^{2}} \frac{1}{1+i \frac{1}{\tau_{D} \omega}}
\end{aligned}
$$

while $C_{0}$ and $g$ are, respectively, the amplitude and the critical frequency of the Lorentz oscillator, $\Gamma$ is the broadening coefficient, $N_{d}$ is the dopant density, $N_{\mathrm{e}-\mathrm{h}}$ is the carrier density, $\tau_{D}$ is the Drude damping time, $m_{\text {opt }}$ is the optical mass of carriers, $\mu$ is the pole order, and $\beta$ is the phase factor of the pole. The rest of the parameters have their usual meaning.

Introducing the dependence of the model parameters on doping density and on the free electron gas density allows to predict the optical properties of Si for any doping density. In the $1.5-5.5 \mathrm{eV}$ spectral range, we found three characteristic oscillator energies sufficient, at energies $E_{g 1}=3.4 \mathrm{eV}, E_{g 2}=3.62 \mathrm{eV}$ and $E_{g 3}=4.27 \mathrm{eV}$, where $E_{g 1}$ and $E_{g 3}$ correspond to the first and second direct transitions in $\mathrm{Si}$, while $E_{g 2}$ is the established energy for $\mathrm{e}-\mathrm{h}$ pair creation in $\mathrm{Si}$ [68]. This is also coherent with literature data [11-13]. Also, the integration of the Drude part with the HMO approximation allows discrimination between the changes in dielectric function for lower energies, since the Drude-dependent influence area (hence connected with free carriers) is localized mainly on the left-hand side of the spectrum and an increase in e- $h$ pair density always influences the lower energies first. On the other hand, the lattice disturbance caused by doping itself is present mainly at the characteristic transition energies $E_{g}$ and neighbouring area.

\subsection{Evolution of parameters with variation of doping concentration}

The experimental results of JELLISON et al. [1 and private communications] for heavily doped Si:P were used to determine model parameters versus the doping density. The dielectric function was predicted on the basis of $[12,69]$ and on the well-known effects originating from doping. In general, the oscillator amplitude $C_{0}$ decreases with 
increasing doping concentration, because of the additional defects and lattice disturbances caused by doping. The broadening parameter $\Gamma$ tends to increase for the same reasons. The critical point energy $E_{g}$ (or frequency $\omega_{g}$ ) shows a slight shift towards lower energies due to the renormalization of the band structure caused by additional free-carrier population originated from doping and state-and-band filling (see Fig. 1).
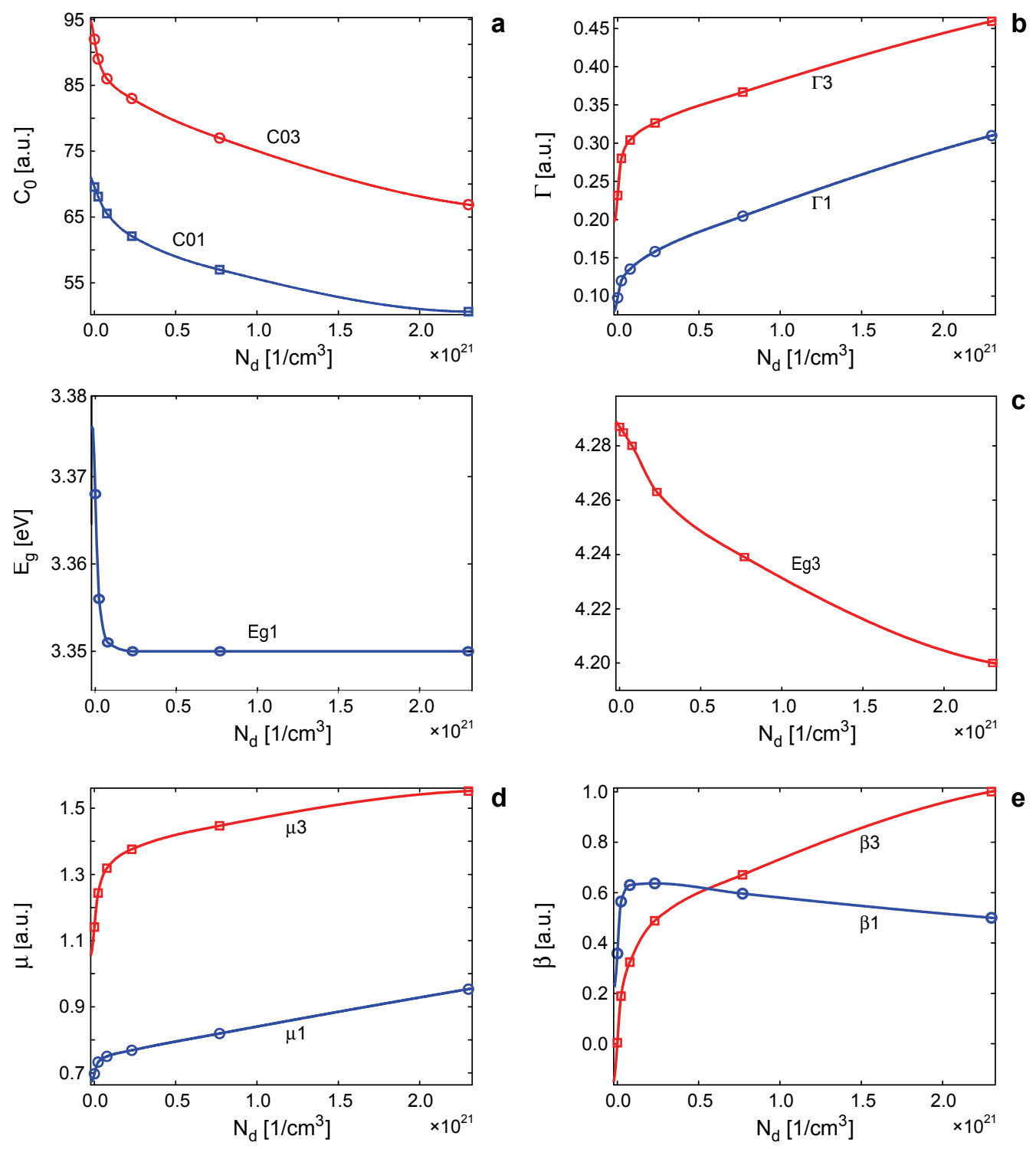

Fig. 1. Evolutions of oscillator amplitude $C_{0}$, broadening parameter $\Gamma$ and critical point energy $E_{g}$ versus doping density are presented on graphs $(\mathbf{a}),(\mathbf{b})$ and $(\mathbf{c})$, respectively. Below the changes of the pole order $|\mu|(\mathbf{d})$ and the pole phase factor $\beta$ (e) versus doping concentration are presented. Tendency lines were obtained by calculating a piece-wise polynomial for each pair of points. 
$\mathrm{T}$ a b l e 1. Parameters used in the three-oscillator model, for five different doping concentrations $N_{d}$. Also goodness of fit $(R)$ and sum of squared errors (SSE) are presented for each doping density.

\begin{tabular}{lllllll}
\hline & \multicolumn{5}{c}{ Doping concentration $N_{d}\left[\mathrm{~cm}^{-3}\right]$} \\
\cline { 2 - 6 } Parameters & $1 \times 10^{14}$ & $2.30 \times 10^{19}$ & $7.70 \times 10^{19}$ & $2.30 \times 10^{20}$ & $7.70 \times 10^{20}$ & $2.30 \times 10^{21}$ \\
\hline$C_{1}$ & 69.56 & 68.1 & 65.53 & 62.1 & 57 & 50.62 \\
$C_{2}$ & 239.3 & 316.5 & 343 & 335.7 & 325.3 & 311.9 \\
$C_{3}$ & 92 & 89 & 86 & 83 & 77 & 66.88 \\
$\gamma_{1}$ & 0.09775 & 0.12 & 0.1353 & 0.1582 & 0.2045 & 0.3098 \\
$\gamma_{2}$ & 0.361 & 0.345 & 0.3408 & 0.3396 & 0.346 & 0.381 \\
$\gamma_{3}$ & 0.2316 & 0.2801 & 0.3042 & 0.3264 & 0.3668 & 0.4594 \\
$\beta_{1}$ & 0.3582 & 0.5648 & 0.63 & 0.6465 & 0.596 & 0.5 \\
$\beta_{2}$ & 0.307 & 0.089 & 0.03906 & 0.03642 & 0.06536 & 0.1675 \\
$\beta_{3}$ & 0.004174 & 0.1891 & 0.3238 & 0.4878 & 0.6708 & 1.001 \\
$\left|\mu_{1}\right|$ & 0.6976 & 0.7328 & 0.7501 & 0.7685 & 0.8193 & 0.9533 \\
$\left|\mu_{2}\right|$ & 0.4398 & 0.3149 & 0.2966 & 0.3108 & 0.3331 & 0.3743 \\
$\left|\mu_{3}\right|$ & 1.141 & 1.244 & 1.319 & 1.376 & 1.447 & 1.552 \\
$E_{g 1}$ & 3.368 & 3.356 & 3.351 & 3.35 & 3.35 & 3.35 \\
$E_{g 2}$ & 3.654 & 3.621 & 3.604 & 3.592 & 3.606 & 3.636 \\
$E_{g 3}$ & 4.287 & 4.285 & 4.28 & 4.263 & 4.239 & 4.2 \\
$R$-square & 0.9998 & 0.9999 & 0.9999 & 0.9999 & 0.9999 & 0.9997 \\
SSE & 1.0909 & 2.2556 & 1.4831 & 0.9643 & 0.8062 & 0.6568 \\
\hline
\end{tabular}

It has been stated before that the condition $\mu<1$ is necessary to satisfy Kramers-Kronig relations [11] and we expect the pole order $\mu$ to decrease as the doping density increases since the associated oscillator strength becomes relatively weaker while the phase factor $|\beta|$ should increase with doping concentration due to the increased fraction of Brillouin zone over which band transitions take place. As can be seen in Fig. 1, the parameter $|\mu|$ varies similarly with the corresponding broadening coefficient $\Gamma$.

Parameters corresponding to an oscillator with critical energy $E_{g} \approx 3.62 \mathrm{eV}$ have a slightly different behavior than predicted. This might be due to additional mechanisms that occur at this transition that are not present for transitions at $E_{g}=$ $=3.38$ and $4.29 \mathrm{eV}$. Parameters of the three-oscillator model (for five different doping densities) are listed in Table 1.

\section{Results}

\subsection{Heavily P-doped Si}

The model reproduces the experimental results nearly perfectly for doped $\mathrm{Si}$ in the energy range $E=2.5-5.0 \mathrm{eV}$. The real and imaginary parts of $\varepsilon$ reproduced by our model and the experimental data of [1] are presented in Figs. $2 \mathbf{a}$ and $2 \mathbf{b}$, 
respectively. For $E=1.2-2.5 \mathrm{eV}$, the imaginary part of the $\varepsilon$ function is very small, except for extremely high P-doping densities, leading to relatively significant differences between the experimental and simulated data. The residual plot of the imaginary component of the dielectric function, shown in Fig. 2c, confirms the fact that the greatest relative differences occur for low energies, but in any case the errors are one order smaller than systematic differences observed on experimentally measured dielectric functions of $\mathrm{Si}$ [13]. Figure 2c confirms the quality of our model. Parameterization of Eq. (1) allowed us to simulate the dielectric functions for any possible P-doping density measured so far for $\mathrm{Si}$, which is presented in Fig. 2d. With the help of Eq. (1), the dielectric function can be calculated for any doping density between $N_{d}=10^{14}$ to $2.3 \times 10^{21} \mathrm{~cm}^{-3}$, where the first corresponds to intrinsic, float-zone $\mathrm{Si}$ and the last is the solubility level of $\mathrm{P}$ in $\mathrm{Si}$.

The absolute values of $\varepsilon$ around the two main energies $E=3.38 \mathrm{eV}$ and $E=4.29 \mathrm{eV}$ decrease with doping concentration. This effect is due to the lattice disturbance caused by defects.
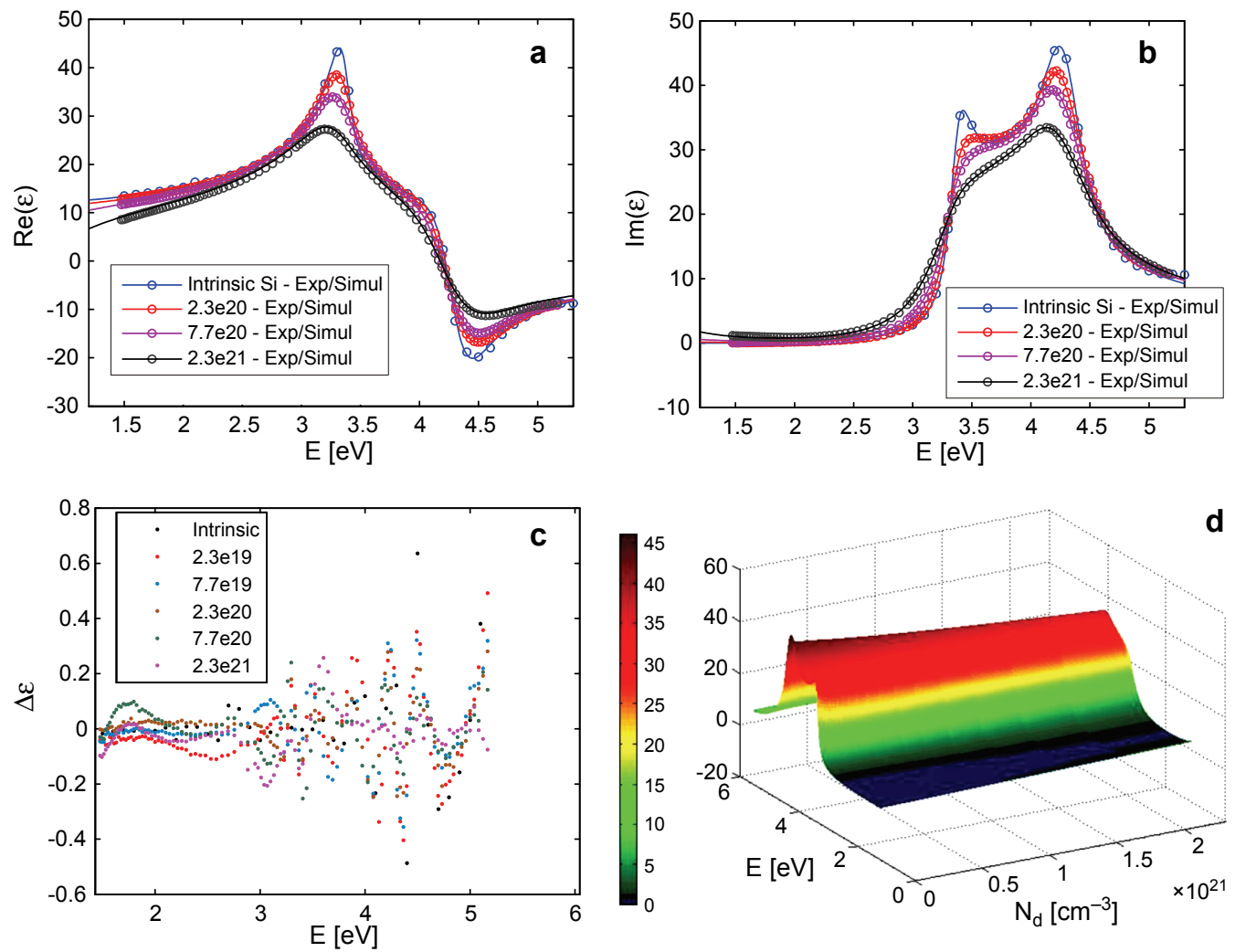

Fig. 2. Simulated (solid line) and experimental (points) data for different P-doping concentrations are shown: $\operatorname{Re}(\varepsilon)(\mathbf{a}), \operatorname{Im}(\varepsilon)(\mathbf{b})$, residual plot for the imaginary part of the dielectric function $\Delta \varepsilon(\mathbf{c})$ and $\operatorname{Im}(\varepsilon)$ of Si for all possible P-doping densities (d). 


\subsection{Highly excited $\mathrm{Si}$}

The model presented is able to determine changes in the dielectric function independently of the lattice disturbance, i.e., in the case of injection or excitation of the dense free-carrier gas. As can be seen in Fig. 3a, the influence of free-carriers on the dielectric function appears mainly at low energies, being negligible in the range around the direct transition peak at $E=3.4 \mathrm{eV}$ and above, where the most important role is played by lattice disturbances and effects introduced by doping. In Figure 3a, one can clearly see that the free-carrier related Drude part of the dielectric function, that is present for energies $E=1$ to $3 \mathrm{eV}$ for the densities studied, is mainly responsible
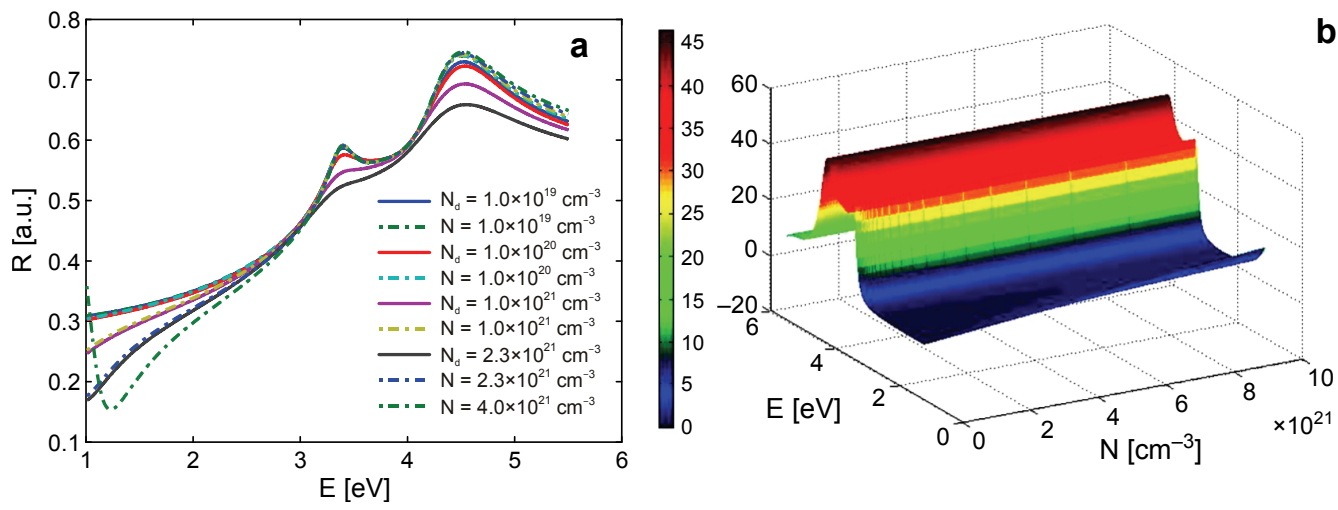

Fig. 3. Simulated reflectivity $R$ for different P-doping concentrations (solid curves) and corresponding density of excited free carriers (dot-dashed curves) (a). Simulated response of $\operatorname{Im}(\varepsilon)$ for free-carrier densities covering the range from intrinsic to near-metallic (b).

for the changes of reflectivity, but it has little or no meaning around the main transition peaks. For the highest presented density $N=4 \times 10^{21} \mathrm{~cm}^{-3}$, one can observe the appearance of the so-called plasma minimum around $E=1.1 \mathrm{eV}$. This minimum in reflectivity depends on carrier density $N$, carrier optical mass $m_{\text {opt }}$ and carrier damping time $\tau$ and tends to move towards higher energies when $N$ increases and/or $\tau$ and $m_{\text {opt }}$ decrease. For higher free-carrier densities, ultimately near-metallic values, the reflectivity reaches values close to unity. One has to keep in mind that the existence and measurement of such high carrier densities in $\mathrm{Si}$ is possible only on a femtosecond-time scale $[70,71]$. That poses additional very complex problems, that we have addressed elsewhere [72].

\section{Conclusions}

The main objective of this work has been successfully met with an excellent precision. The model determines the dielectric functions over the complete P-doping range, from intrinsic to extremely highly doped $\mathrm{Si}$ (up to $2.3 \times 10^{21} \mathrm{~cm}^{-3}$ ), thanks to a parameterization of tendency lines for variables of Eq. (1), which were obtained by 
analysis of experimental results of ellipsometrically measured dielectric functions of Si:P. The Drude-Lorentz parameters describe the optical functions of lightly or moderately doped $\mathrm{Si}$ in the corresponding full range of free-carrier densities up to a metal-like density. The results are consistent with physical predictions and previous works [1-4]. A clear distinction between optical functions of Si with an injected free-carrier population and one originating from doping could be established. Future work shall include the effects of the temperature on the dielectric function of $\mathrm{Si}$ and extension to other semiconductors and dielectrics.

Simulated optical functions (reflectivity, absorption coefficient) agree with experimental results published previously $[73,74]$ and obtained by us on MINDs with extremely high steady-state free-carrier densities.

Acknowledgements - the authors would like to thank The Regional Government of Lower Silesia (Poland) for financial support.

\section{References}

[1] Jellison G.E., JR., Withrow S.P., McCamy J.W., Budai J.D., Lubben D., Godbole M.J., Optical functions of ion-implanted, laser-annealed heavily doped silicon, Physical Review B 52(20), 1995, pp.14607-14614

[2] Aspnes D.E., Studna A.A., Dielectric functions and optical parameters of Si, Ge, GaP, GaAs, GaSb, InP, InAs and InSb from 1.5 to $6.0 \mathrm{eV}$, Physical Review B 27(2), 1983, pp. 985-1009.

[3] Fried M., Lohner T., Aarnink W.A.M., Hanekamp L.J., van Silfhout A., Determination of complex dielectric functions of ion implanted and implanted-annealed amorphous silicon by spectroscopic ellipsometry, Journal of Applied Physics 71(10), 1992, pp. 5260-5262.

[4] PIng Sheng, Theory for the dielectric function of granular composite media, Physical Review Letters 45(1), 1980, pp. 60-63.

[5] Olevano V., Palummo M., Onida G., del Sole R., Exchange and correlation effects beyond the LDA on the dielectric function of silicon, Physical Review B 60(20), 1999, pp. 14224-14233.

[6] Green M.A., Third generation photovoltaics: solar cells for 2020 and beyond, Physica E 14(1-2), 2002, pp. 65-70.

[7] Aspnes D.E., Quinn W.E., Tamargo M.C., Pudensi M.A.A., Schwarz S.A., Brasil M.J.S.P., NAHORY R.E., GREGORY S., Growth of $A l_{x} G a_{1-x}$ As parabolic quantum wells by real-time feedback control of composition, Applied Physics Letters 60(10), 1992, pp. 1244-1246.

[8] Peters M., Rüdiger M., Hermle M., Blaesi B., Photonic crystals in solar cells: a simulation approach, Proceedings of SPIE 7725, 2010, article 772514.

[9] Honsberg C.B., Yun F., Green M.A., Wenham S.R., Mechanically grooved, multi-junction, interdigitated rear contact silicon solar cell for low lifetime substrates, 12th European Photovoltaic Solar Energy Conference, April, 1994, pp. 63-66.

[10] Toigo F., Woodruff T.O., Calculation of the dielectric function for a degenerate electron gas with interactions. I. Static limit, Physical Review B 2(10), 1970, pp. 3958-3966.

[11] Leng J., Opsal J., Chu H., Senko M., Aspnes D.E., Analytic representations of the dielectric functions of materials for device and structural modeling, Thin Solid Films 313-314, 1998, pp. 132-136.

[12] Jellison G.E., JR., Modline F.A., Parametrization of the optical functions of amorphous materials in the interband region, Applied Physics Letters 69(3), 1996, pp. 371-373.

[13] Bell K.A., Mantese L., Rossow U., Aspnes D.E., Systematic differences among nominal reference dielectric function spectra for crystalline Si as determined by spectroscopic ellipsometry, Thin Solid Films 313-314, 1998, pp. 161-166. 
[14] Glunz S.W., High-efficiency crystalline silicon solar cells, Advances in Optoelectronics 2007, pp. $1-15$.

[15] Kuznicki Z.T., Bigot J.-Y., Optically active Si surfaces, Proceedings of SPIE 7002, 2008, article 70020R.

[16] Smith D.R., Padilla W.J., Vier D.C., Nemat-Nasser S.C., Schultz S., Composite medium with simultaneously negative permeability and permittivity, Physical Review Letters 84(18), 2000, pp. 4184-4187.

[17] Hwang E.H., Das Sarma S., Dielectric function, screening and plasmons in two-dimensional graphene, Physical Review B 75(20), 2007, article 205418.

[18] Bender M., Trube J., Stollenwerk J., Deposition of transparent and conducting tindium-tin-oxide films by the r.f.-superimposed DC sputtering technology, Thin Solid Films 354(1-2), 1999, pp. 100-105.

[19] Schubert M., Rheinländer B., Woollam J.A., Johs B., Herzinger C.M., Extension of rotating-analyzer ellipsometry to generalized ellipsometry: determination of the dielectric function tensor from uniaxial $\mathrm{TiO}_{2}$, Journal of the Optical Society of America A 13(4), 1996, pp. 875-883.

[20] Chen T.P., Liu Y., Tse M.S., Tan O.K., Ho P.F., Liu K.Y., Gui D., Tan A.L.K., Dielectric functions of Si nanocrystals embedded in a $\mathrm{SiO}_{2}$ matrix, Physical Review B 68(15), 2003, article 153301.

[21] KuZNICKI Z.T., Enhanced absorption and quantum efficiency in locally modified single-crystals Si, Applied Physics Letters 81(25), 2002, pp. 4853-4855.

[22] Ehrenreich H., Philipp H.R., Phillips J.C., Interband transitions in groups 4, 3-5 and 2-6 semiconductors, Physical Review Letters 8(2), 1962, pp. 59-61.

[23] Cardona M., Greenaway D.L., Reflectivity of gray tin single crystals in the fundamental absorption region, Physical Review 125(4), 1962, pp.1291-1296.

[24] Jungk G., Many-particle effects at the $E_{1}$-transition in silicon, Physica Status Solidi B 99(2), 1980, pp. 643-650.

[25] Jellison G.E., JR., Modine F.A., Optical functions of silicon between 1.7 and $4.7 \mathrm{eV}$ at elevated temperatures, Physical Review B 27(12), 1983, pp. 7466-7472.

[26] SARI S.O., SchNatterly S.E., Optical spectroscopy of semiconductors in high magnetic fields using polarization modulation, Surface Science 37, 1973, pp. 328-339.

[27] Matatagui E., Thompson A.G., Cardona M., Thermoreflectance in semiconductors, Physical Review 176(3), 1968, pp. 950-960.

[28] Guizzetti G., Nosenzo L., Reguzzoni E., Samoggia G., Thermoreflectance spectra of diamond and zinc-blende semiconductors in the vacuum-ultraviolet region, Physical Review B 9(2), 1974, pp. 640-647.

[29] Daunois A., Aspnes D.E., Electroreflectance and ellipsometry of silicon from 3 to $6 \mathrm{eV}$, Physical Review B 18(4), 1978, pp. 1824-1839.

[30] Grover J.W., Handler P., Electroreflectance of silicon, Physical Review B 9(6), 1974, pp. 2600-2606.

[31] Zucca R.R.L., Shen Y.R., Wavelength-modulation spectra of some semiconductors, Physical Review B 1(6), 1970, pp. 2668-2676.

[32] Welkowsky M., Braunstein R., Interband transitions and exciton effects in semiconductors, Physical Review B 5(2), 1972, pp. 497-509.

[33] KuZNicki Z.T., Ley M., Enhanced absorbance of a strained nanoscale Si-layered system, Applied Physics Letters 82(24), 2003, pp. 4241-4243.

[34] Sze S.M., Physics of Semiconductor Devices, John Wiley and Sons, 1981.

[35] Lautenschlager P., Garriga M., Vina L., Cardona M., Temperature dependence of the dielectric function and interband critical points in silicon, Physical Review B 36(9), 1987, pp. 4821-4830.

[36] BASU S., LeE B.J., ZhANG Z.M., Infrared radiative properties of heavily doped silicon at room temperature, Journal of Heat Transfer 132(2), 2010, article 023301.

[37] Benedict L.X., Wethkamp T., Wilmers K., Cobet C., Esser N., Shirley E.L., Richter W., Cardona M., Dielectric function of wurtzite GaN and AlN thin films, Solid State Communications 112(3), 1999, pp. 129-133. 
[38] Goldhahn R., Schley P., Winzer A.T., Gobsh G., Cimalla V., Ambacher O., Rakel M., Cobet C., EsSer N., Lu H., Schaff W.J., Detailed analysis of the dielectric function for wurtzite InN and In-rich InAlN alloys, Physica Status Solidi A 203(1), 2006, pp. 42-49.

[39] Li Huang, Callan J.P., Glezer E.N., Mazur E., GaAs under intense ultrafast excitation: response of the dielectric function, Physical Review Letters 80(1), 1998, pp. 185-188.

[40] KuZnicki Z.T., MeyrueIs P., Solar-light induced opacity of MIND cells, Proceedings of SPIE 6197, 2006, article 619716.

[41] Viña L., Logothetidis S., Cardona M., Temperature dependence of the dielectric function of germanium, Physical Review B 30(4), 1984, pp. 1979-1991.

[42] Logothetidis S., Cardona M., Lautenschlager P., Garriga M., Temperature dependence of the dielecric function and the interband critical points of CdSe, Physical Review B 34(4), 1986, pp. 2458-2469.

[43] Y IN Z., Sмiтн F.W., Optical dielectric functions and infrared absorption of hydrogenated amorphous silicon nitride films: experimental results and effective-medium-approximation analysis, Physical Review B 42(6), 1990, pp. 3666-3675.

[44] Kuznicki Z.T., Ley M., Lezec H.J., Sarrabayrouse G., Rousset B., Rossel F., Migeon H., Wirtz T., Non-linear optical functions of crystalline-Si resulting from nanoscale layered systems, Materials Science and Engineering C 26(5-7), 2006, pp. 961-965.

[45] De Filippo F., de Lisio C., Maddalena P., Lérondel G., Yao T., Altucci C., Determination of the dielectric function of porous silicon by high-order laser-harmonic radiation, Applied Physics A 73(6), 2001, pp. 737-740.

[46] THEISs W., The dielectric function of porous silicon - how to obtain it and how to use it, Thin Solid Films 276(1-2) ,1996, pp. 7-12.

[47] Lioudakis E., Nassiopoulou A., Отноnos A., Ellipsometric analysis of ion-implanted polycrystalline silicon films before and after annealing, Thin Solid Films 496(2), 2006, pp. 253-258.

[48] Aspnes D.E., Theeten J.B., Dielectric function of $\mathrm{Si}-\mathrm{SiO}_{2}$ and $\mathrm{Si}_{-} \mathrm{Si}_{3} \mathrm{~N}_{4}$ mixtures, Journal of Applied Physics 50(7), 1979, pp. 4928-4935.

[49] Penn D.R., Wave-number-dependent dielectric function of semiconductors, Physical Review 128(5), 1962, pp. 2093-2097.

[50] Bertsch G.F., Inata J.-I., Rubio A., Yabana K., Real-space, real-time method for the dielectric function, Physical Review B 62(12), 2000, pp. 7998-8002.

[51] Kim C.C., Garland J.W., Abad H., Raccah P.M., Modeling the optical dielectric function of semiconductors: Extension of the critical-point parabolic-band approximation, Physical Review B 45(20), 1992, pp. 11749-11767.

[52] Penn D.R., Electron mean-free-path calculations using a model dielectric function, Physical Review B 35(2), 1987, pp. 482-486.

[53] SRinivasan G., Microscopic dielectric function of a model semiconductor, Physical Review 178(3), 1969, pp. 1244-1251.

[54] TAYLOR R., A simple, useful analytical form of the static electron gas dielectric function, Journal of Physics F: Metal Physics 8(8), 1978, pp. 1699-1702.

[55] Ichimaru S., Utsumi K., Analytic expression for the dielectric screening function of strongly coupled electron liquids at metallic and lower densities, Physical Review B 24(12), 1981, pp. 7385-7388.

[56] Spanier J.E., Herman I.P., Use of hybrid phenomenological and statistical effective-medium theories of dielectric functions to model the infrared reflectance of porous SiC films, Physical Review B 61(15), 2000, pp. 10437-10450.

[57] Kootstra F., De Boeij P.L., Snijders J.G., Application of time-dependent density-functional theory to the dielectric function of various non-metallic crystals, Physical Review B 62(11), 2000, pp. 7071-7083.

[58] Levine Z.H., Louie S.G., New model dielectric function and exchange-correlation potential for semiconductors and insulators, Physical Review B 25(10), 1982, pp. 6310-6316.

[59] Yu P.Y., Cardona M., Fundamentals of Semiconductors, Springer-Verlag, Berlin, 1996. 
[60] Harrison W.A., Electronic Structure and the Properties of Solids: The Physics of Chemical Bonds, W.H. Freeman and Company, San Francisco, 1980.

[61] Ridley B.K., Quantum Processes in Semiconductors, Clarendon Press, Oxford, 1998.

[62] Cohen M.L., Chelicowsky J.R., Electronic Structure and Optical Parameters of Semi-Conductors, Springer Series in Solid-State Sciences, Springer-Verlag, Berlin, 1981.

[63] Lanoo M., Bourgoin J., Points Defects in Semiconductors I, Theoretical Aspects, Springer-Verlag, Berlin, 1981.

[64] Weckebach W.T., Essentials of Semiconductor Physics, John Wiley and Sons, Chichester, 1999.

[65] Walter J.P., Cohen M.L., Wave-vector-dependent dielectric function for Si, Ge, GaAs and ZnSe, Physical Review B 2(6), 1970, pp. 1821-1826.

[66] Yu G., Soga T., Jimbo T., Umeno M., Characterization of MOCVD-grown GaAs on Si by spectroscopic ellipsometry, Applied Surface Science 100-101, 1996, pp. 617-620.

[67] SoKolowski-TinTen K., vON DER Linde D., Generation of dense electron-hole plasmas in silicon, Physical Review B 61(4), 2000, pp. 2643-2650.

[68] Canfield L.R., Kerner J., Korde R., Stability and quantum efficiency performance of silicon photodiode detectors in the far ultraviolet, Applied Optics 28(18), 1989, pp. 3940-3943.

[69] ERman M., Andre J.P., LeBris J., Spectroscopic ellipsometry study of InP, GaInAs, GaInAs/P heterostructures, Journal of Applied Physics 59(6), 1986, pp. 2019-2025.

[70] Huber R., Tauser F., Brodschelm A., Bichler M., Abstreiter G., Leitenstorfer A., How many-particle interactions develop after an ultrafast excitation of an electron-hole plasma, Nature 414(6861), 2001, pp. 286-289.

[71] Altukhov P.D., Kuzminov E.G., The self-compression of injected electron-hole plasma in silicon, Physica Status Solidi B 232(2), 2002, pp. 364-379.

[72] Sieradzki A., Basta M., Bigot J.-Y., KuźNicki Z.T., Ultrafast excitation of dense electron gas in silicon nanostructures, (to be published).

[73] BüCher K., BRuns J., Wagemann H.G., Absorption coefficient of silicon: an assessment of measurements and the simulation of temperature variation, Journal of Applied Physics 75(2), 1994, pp. 1127-1132.

[74] Aspnes D.E., Studna A.A., KinsBron E., Dielectric properties of heavily doped crystalline and amorphous silicon from 1.5 to $6.0 \mathrm{eV}$, Physical Review B 29(2), 1984, pp. 768-779.

Received October 19, 2011

in revised form February 21, 2012 